\title{
DESEMPENHO DE CULTIVARES DE ALFACE CRESPA PRODUZIDAS EM HIDROPONIA A PARTIR DE MUDAS PRODUZIDAS EM FLOATING E ESPUMA FENÓLICA
}

\author{
Ronan Gualberto, Gabriela Lara Leite Alcalde, Carolina Lembe Silva \\ Universidade de Marília - UNIMAR, Curso de Engenharia Agronômica, Marília, SP. E-mail: ronangual@hotmail.com
}

\begin{abstract}
RESUMO
Objetivando-se avaliar o desempenho de cultivares de alface crespa produzidas em hidroponia a partir de mudas produzidas em floating e espuma fenólica, realizou-se um experimento em casa de vegetação, na Fazenda Experimental da Universidade de Marília-SP. O delineamento experimental adotado foi o inteiramente casualizado em esquema fatorial $2 \times 2$, com cinco repetições, cujos fatores corresponderam respectivamente, a dois sistemas de cultivo para a produção de mudas (floating e espuma fenólica) e duas cultivares de alface crespa (Jullie e TPC). Foram avaliadas as características massa fresca e seca da parte aérea, número de folhas por planta e comprimento do caule. Ocorreram diferenças significativas entre cultivares para todos os caracteres avaliados. Diferença estatística significativa entre sistemas de cultivos foi observada somente para o comprimento de caule. A interação cultivar $\mathrm{x}$ sistemas de cultivos não foi significativa para nenhum dos caracteres avaliados. No número de folhas por planta a cultivar TPC foi superior a "Jullie" independentemente do sistema de cultivo. Já para o comprimento de caule, constatouse que as duas cultivares apresentaram valores superiores no sistema floating em relação à espuma fenólica e que a cultivar Jullie apresentou comprimento de caule maior que a "TPC" em ambos os sistemas. Esses valores mais altos são indesejáveis, uma vez que são responsáveis pelo pendoamento precoce na alface. Para a massa seca da parte aérea verificou-se que a cultivar Jullie foi superior a "TPC" na espuma fenólica, porém não ocorreu diferença entre elas no sistema floating. Conclui-se que nas condições em que foi conduzido o experimento que a cultivar Jullie apresentou maior massa fresca da parte aérea do que a cultivar TPC e que no número de folhas a "TPC" se destacou em relação à "Jullie" e que os sistemas de cultivos não influenciaram no rendimento das cultivares.
\end{abstract}

Palavras-chave: ambiente protegido; cultivo sem solo; Lactuca sativa L.; rendimento; sistemas de cultivo.

\section{PERFORMANCE OF CRISPY LETTUCE CULTIVARS GROWN UNDER THE HIDROPONIC SYSTEM USING SEEDLINGS PRODUCED IN FLOATING AND PHENOLIC FOAM}

\begin{abstract}
With the purpose to evaluate the performance of crispy lettuce cultivars grown under the hidroponic system using seedlings produced in floating and phenolic foam was carry out this experiment in the greenhouse of Experimental Farm, of Marília University. The experimental design was a completely randomized arranged in a factorial scheme $2 \times 2$ with five replicates, whose factors corresponded respectively to two cultivation systems for the production of seedlings (floating and phenolic foam) and two cultivars of crisp lettuce ( Julie and TPC). The characteristics fresh and dry mass of the shoot, number of leaves per plant and stem length were evaluated. There were significant differences between cultivars for all evaluated traits. Significant statistical difference between cropping systems was observed only for stem length. The interaction cultivar $x$ cropping systems was not significant for any of the characters evaluated. In the number of leaves per plant TPC cultivar was superior to "Jullie" regardless of the culture system. As for stem length, it was found that the two cultivars showed higher values in the floating system in relation to the phenolic foam and that the Jullie cultivar had a longer stem length than the "TPC" in the phenolic foam. These higher values are undesirable, as they are responsible for early tillage in lettuce. For the dry mass of the aerial part, it was verified that the cultivar Jullie was superior to "TPC" in the phenolic foam, but did not occur difference between them in the floating system. It was concluded that under the conditions in which the experiment was conducted, the Jullie cultivar showed a higher fresh shoot biomass
\end{abstract}


than the TPC cultivar and that in the number of leaves the "TPC" stood out in relation to "Jullie" and that the systems did not influence the yield of the cultivars.

Keywords: cropping systems; Lactuca sativa L.; protected cultivation; soilless cultivation; yield.

\section{INTRODUÇÃO}

A alface (Lactuca sativa L.) é uma hortaliça folhosa bastante consumida em todo o mundo e a de maior expressão econômica no Brasil. É consumida de forma in natura, sendo boa fonte de vitaminas e sais minerais e devido ao baixo teor de calorias, recomendada para dietas alimentares ricas em fibras (FILGUEIRA, 2008). Em algumas centrais de distribuição, o conjunto das espécies de alface representa quase $50 \%$ de todas as folhosas que são comercializadas e, dentre essas, a crespa corresponde a quase $40 \%$ do total (MORETTI; MATTOS, 2006).

Devido às mudanças no hábito alimentar do consumidor que vem preocupando-se mais com a saúde, o consumo de alface vem aumentando a cada ano sendo necessária sua produção diária durante todo o ano para atender a crescente demanda do mercado consumidor com mercadoria fresca e com qualidade. Desta maneira, nos últimos anos têm sido desenvolvidos e adotados sistemas de cultivo protegido (SOUZA et al., 1994), principalmente o hidropônico.

O cultivo de hortaliças em ambiente protegido é bastante difundido e aceito nas áreas de produção em todo o país. A sua aceitação e expansão entre produtores deve-se à exploração racional de pequenas áreas e à garantia de colheita, permitindo a obtenção de produções elevadas e de melhor qualidade (QUEIROZ et al., 2004). Em decorrência da sensibilidade da planta às intempéries e às variações climáticas, o cultivo da alface em ambiente protegido vem crescendo em importância nos últimos anos. Além da praticidade no manejo, a limpeza e a versatilidade desta modalidade de cultivo conferem ótimas condições para reduções na utilização de produtos químicos, menor consumo de água, produção fora de época, maior produtividade $e$, consequentemente, melhor preço, devido à alta qualidade do produto (CASTELLANE; ARAÚJO, 1994; FAQUIN et al., 1996; RESH, 1997; PAIVA, 1998).

O sucesso de uma cultura depende, em grande parte, da utilização de mudas de alta qualidade, seja no caso de frutífera, olerícola ou florestal. Os principais fatores envolvidos na formação de uma muda são o substrato, a nutrição e o recipiente, os quais devem proporcionar um bom desenvolvimento da muda durante a sua permanência no viveiro, tendo em vista a sua importância no desempenho da futura planta. O substrato exerce a função do solo, fornecendo à planta sustentação, nutrientes, água e oxigênio. Os substratos podem ter diversas origens, ou seja, animal (esterco, húmus, etc.), vegetal (tortas, bagaços, xaxim, serragem, etc.), mineral (vermiculita, perlita, areia, etc.) e artificial (espuma fenólica, isopor, etc.).

$\mathrm{Na}$ produção de mudas, para o cultivo sem solo de olerícolas, deve-se objetivar produzir plantas suficientemente desenvolvidas, com o fim de diminuir o período entre o transplante e a colheita, garantindo, desta maneira, uma otimização da mão de obra

disponível e da área da estufa de cultivo definitivo, bem como, um ótimo índice de estabelecimento da população e uma boa sustentação da muda no sistema de cultivo sem solo. Este novo paradigma exige mudança no sistema de produção quanto aos

materiais empregados como substratos (HORNER et al., 2003). Ainda segundo esses autores a escolha do substrato determinará o tipo de estrutura e o manejo da irrigação para a produção das mudas. Assim, existe necessidade de estudar sistemas específicos para a produção de mudas a serem utilizadas no cultivo sem solo.

Segundo Furlani et al. (2009) existem quatro principais tipos de mudas para hortaliças folhosas, como a alface que têm sido usadas no cultivo hidropônico, à saber: substrato organomineral, vermiculita, algodão hidrófilo, fibra de coco e espuma fenólica. Os sistemas de piscina (floating) e recentemente, a espuma fenólica têm sido os mais empregados para a produção de mudas de oleráceas folhosas para a hidroponia.

O sistema denominado de Floating (flutuante) ou piscina tem sido muito utilizado para a produção de mudas de alface, e consiste 
em se colocar bandejas com substrato em piscinas contendo água, adicionada de solução nutritiva (hidroponia) ou não; e estas servirão como suprimento de água (BORNER, 1999). Com isso, diminui a preocupação do olericultor em manter sempre úmido os substratos com sistemas de irrigação tradicionais (aspersão ou rega manual) além de garantir a obtenção de mudas de boa qualidade.

Segundo Furlani et al. (2009) a espuma fenólica é um substrato estéril, de fácil manuseio e que oferece ótima sustentação para as plântulas, reduzindo sensívelmente os danos durante a operação de transplantio. Dispensa o uso de bandejas de isopor, portanto não requer a construção do "floating", pois após a emergência as mudas são transplantadas diretamente para os canais de crescimento. É comercializado em placas com $2 \mathrm{~cm}$ ou $4 \mathrm{~cm}$ de espessura e com células pré-marcadas nas dimensões de $2 \mathrm{~cm} \times 2$ $\mathrm{cm}$.

Diante do exposto, a hipótese desta pesquisa é que os diferentes substratos utilizados na produção das mudas podem influenciar no desempenho das cultivares de alface, produzidas em hidroponia. Assim sendo, o presente trabalho teve o objetivo de avaliar o desempenho de cultivares de alface crespa produzidas em hidroponia a partir de mudas produzidas em floating e espuma fenólica.

\section{MATERIAL E MÉTODOS}

O Experimento foi conduzido sob casade-vegetação na Fazenda Experimental da Universidade de Marília - SP, com altitude de 610 $\mathrm{m}$, latitude $22^{\circ} 12^{\prime} 50^{\prime \prime} \mathrm{S}$ e longitude $49^{\circ} 56^{\prime} 45^{\prime \prime}$ W, no período de 17 de março a 19 de maio de 2014.

A estufa utilizada foi do tipo túnel alto, com 4,0 $\mathrm{m}$ de pé direito, cobertura plástica de 150 micras de espessura e laterais protegidas com telas de polipropileno preto com $70 \%$ de sombreamento. Foram utilizadas duas cultivares de alface do grupo crespa: Jullie e TPC e dois sistemas de cultivo na produção das mudas: floating e espuma fenólica. $O$ delineamento experimental adotado foi 0 inteiramente casualizado, com cinco repetições, em esquema fatorial $2 \times 2$. Foi utilizada a técnica do fluxo laminar de nutrientes (NFT = Nutrient Film Technique ), que consiste em manter $2 / 3$ do sistema radicular imerso num filme de solução nutritiva mantido através de fluxo intermitente, obtido com o uso de um "Timer" mecânico, que acionava e desligava a moto bomba a intervalos de tempo pré determinados, em função das fases de cultivo.

O experimento constituiu-se de duas fases distintas: produção das mudas e crescimento final das plantas. No sistema de floating as cultivares foram semeadas em bandejas de poliestireno expandido, com 128 células, em vermiculita. Após a emergência (4 dias após semeadura) as bandejas foram colocadas nas piscinas (berçário) com a solução nutritiva recirculando durante o dia, sendo que no período noturno a moto bomba permanecia desligada. Nesta fase a condutividade elétrica foi mantida entre 1,0 e $1,5 \mathrm{mS} \mathrm{cm}^{-1}$.

No sistema de espuma fenólica, as cultivares foram semeadas em placas de 2,0 × 2,0 $\mathrm{cm}$, após serem lavadas em água corrente. Após a emergência das plântulas as mesmas foram irrigadas diariamente com a mesma solução utilizada no floating por 15 dias, após o qual as células foram destacadas das placas e colocadas na fase de pré crescimento.

No caso do floating após 27 dias da semeadura as mudas foram retiradas das bandejas e as raízes lavadas para retirada da vermiculita, após o qual foram transplantadas para as bancadas de pré crescimento. Nessas bancadas, com perfis trapezoidais feitos de polipropileno com dimensões indicadas para a fase de crescimento inicial, as plantas permaneceram durante 14 dias. Neste ponto, as plantas foram transplantadas para a fase final.

$\mathrm{Na}$ fase de crescimento final as bancadas com 12,0 m de comprimento, 2,0 m de largura e $2 \%$ de declividade eram de tubos de PVC $(75 \mathrm{~mm})$ perfurados, com diâmetro de $5,0 \mathrm{~cm}$ e espaçados a cada $25 \mathrm{~cm}$. As plantas permaneceram nesta fase 21 dias, não ocorrendo diferenças marcantes no ciclo das cultivares testadas, onde então foram realizadas a colheita e as avaliações das características: comprimento de caule, produção de massa fresca e seca por planta e número de folhas por planta. A solução nutritiva utilizada em todo o ciclo da cultura foi a recomendada por, (BASSO; BERNARDES, 1993), utilizando-se por litro: $236,3 \mathrm{mg}$ de $\mathrm{N}, 39,0 \mathrm{mg}$ de $\mathrm{P}, 224,9 \mathrm{mg}$ de $\mathrm{K}$, $228,0 \mathrm{mg}$ de $\mathrm{Ca}, 33,7 \mathrm{mg}$ de $\mathrm{Mg}, 46,3 \mathrm{mg}$ de $\mathrm{S}$, $0,24 \mathrm{mg}$ de $\mathrm{B}, 0,02 \mathrm{mg}$ de $\mathrm{Cu}, 5,0 \mathrm{mg}$ de $\mathrm{Fe}, 0,23$ $\mathrm{mg}$ de $\mathrm{Mn}, 0,03 \mathrm{mg}$ de Mo e 0,05 mg de Zn. No caso do Ferro, foi utilizado $40 \mathrm{~g}$ por 1.000 litros de solução de ferro EDDHA - Ferrilene. Tanto na fase de pré crescimento como na de crescimento final, diariamente foi feita a reposição do volume 
absorvido e evapotranspirado de solução nutritiva pelas plantas, em função da condutividade elétrica, sendo que, quando através de um condutivímetro, observou-se valores abaixo de $1,7 \mathrm{mS} \mathrm{cm}^{-1}$ era acrescentada solução e quando os valores eram superiores a $2,0 \mathrm{mS} \mathrm{cm}^{-1}$ adicionava-se água. Esses valores foram definidos em função da época de realização do experimento (FURLANI, 1995). Em relação ao $\mathrm{pH}$ da solução, este foi monitorado diariamente para verificar se estava na faixa de 5,5 a 6,5. Como a cada 15 dias era renovada toda a solução do tanque de bombeamento, não houve necessidade de ajuste. A água utilizada era de poço semi artesiano, a qual tinha valor de $\mathrm{pH}$ de 6,8 e condutividade elétrica de $0,0 \mathrm{mS} \mathrm{cm}^{-1}$.
Os dados foram submetidos à análise de variância, pelo programa computacional SISVAR (FERREIRA, 2000), utilizando-se o teste de ScottKnott (1974) a 5\% de probabilidade para comparação das médias.

\section{RESULTADOS E DISCUSSÃO}

Observou-se pelo resumo da análise de variância que ocorreram diferenças significativas entre cultivares para todos os caracteres avaliados. Diferença estatística significativa entre sistemas de cultivos foi observada para o comprimento de caule (CC). A interação cultivar $x$ sistemas de cultivos não foi significativa para nenhum dos caracteres avaliados (Tabela 1).

Tabela 1. Número de folhas por planta (NF), comprimento de caule (CC), massa fresca da parte aérea (MFPA) e massa seca da parte aérea (MSPA) de duas cultivares de alface, com as mudas obtidas em dois sistemas de cultivo (floating e espuma fenólica), no sistema hidropônico 'NFT'. Marília, UNIMAR, 2015.

\begin{tabular}{|c|c|c|c|c|c|c|c|c|}
\hline \multirow[b]{3}{*}{ Sistemas de Cultivo } & \multicolumn{2}{|c|}{ NF } & \multicolumn{2}{|c|}{$\mathrm{CC}(\mathrm{cm})$} & \multicolumn{2}{|c|}{ MFPA (g) } & \multicolumn{2}{|c|}{ MSPA (g) } \\
\hline & \multicolumn{2}{|c|}{ Cultivares } & \multicolumn{2}{|c|}{ Cultivares } & \multicolumn{2}{|c|}{ Cultivares } & \multicolumn{2}{|c|}{ Cultivares } \\
\hline & JULLIE & TPC & JULLIE & TPC & JULLIE & TPC & JULLIE & TPC \\
\hline Floating & $18,00 \mathrm{aB}$ & $25,45 \mathrm{aA}$ & $3,01 \mathrm{aA}$ & $2,73 a \mathrm{~B}$ & $256,50 a$ & $209,75 a$ & $10,38 a A$ & $9,03 a B$ \\
\hline \multirow[t]{3}{*}{ Espuma Fenólica } & $18,85 a \mathrm{~B}$ & $24,40 a A$ & $2,06 \mathrm{bA}$ & $1,41 b B$ & A & $\mathrm{B}$ & $11,80 a A$ & $8,95 \mathrm{aB}$ \\
\hline & & & & & $288,00 a$ & $191,75 a$ & & \\
\hline & & & & & & $\mathrm{B}$ & & \\
\hline $\mathbf{F}_{\text {cultivar }}$ & \multicolumn{2}{|c|}{$31,24^{* *}$} & \multicolumn{2}{|c|}{$6,08^{*}$} & \multicolumn{2}{|c|}{$37,5^{* *}$} & \multicolumn{2}{|c|}{$18,05^{* *}$} \\
\hline $\mathbf{F}_{\text {sistemas }}$ & \multicolumn{2}{|c|}{$0,007^{\text {ns }}$} & \multicolumn{2}{|c|}{$36,19 * *$} & \multicolumn{2}{|c|}{$0,33^{\mathrm{ns}}$} & \multicolumn{2}{|c|}{$1,83^{\mathrm{ns}}$} \\
\hline $\mathbf{F}_{\text {cultivarXsistemas }}$ & \multicolumn{2}{|c|}{$0,66^{\text {ns }}$} & \multicolumn{2}{|c|}{$0,94^{\text {ns }}$} & \multicolumn{2}{|c|}{$4,49^{\text {ns }}$} & \multicolumn{2}{|c|}{$2,29^{\text {ns }}$} \\
\hline CV (\%) & \multicolumn{2}{|c|}{12,00} & \multicolumn{2}{|c|}{18,29} & \multicolumn{2}{|c|}{11,04} & \multicolumn{2}{|c|}{11,00} \\
\hline
\end{tabular}

* Médias seguidas pela mesma letra minúscula na coluna e médias seguidas pela mesma letra maiúscula na linha não diferem entre si pelo teste de Scott-Knott ao nível de $5 \%$ de probabilidade. ${ }^{*} p<0,05 ;{ }^{* *} p<0,01 ;{ }^{\text {ns }}$ não significativo $(p>0,05)$

No número de folhas por planta (NF) a cultivar TPC foi superior a "Jullie" independente do sistema de cultivo. Já para o comprimento de caule, constatou-se que as duas cultivares apresentaram valores superiores no sistema floating em relação à espuma fenólica e que a cultivar Jullie apresentou comprimento de caule maior que a "TPC" na espuma fenólica. Esses valores mais altos são indesejáveis, uma vez que são responsáveis pelo pendoamento precoce da alface. Para a massa seca da parte aérea verificou-se que a cultivar Jullie foi superior a "TPC" na espuma fenólica, porém não ocorreu diferença entre elas no sistema floating (Tabela $1)$.

Para a massa fresca da parte aérea (MFPA) a cultivar Jullie também foi superior à "TPC" independente do sistema de cultivo (Tabela 1).
Avaliando a produção de mudas de alface em floating Souza et al. (2007) concluíram que o sistema não é eficiente para o crescimento das raízes de alface. Já segundo Santin et al. (2005) verificando a relação entre substratos e métodos de aplicação de solução nutritiva na produção de mudas e a posterior resposta produtiva da beterraba observaram que não há diferenças entre fertirrigação e flutuação contínua em solução nutritiva.

Horner et al. (2003) em avaliação do crescimento de mudas para o cultivo sem solo do tomateiro em diferentes substratos verificaram que as plantas produzidas em vermiculita apresentaram valores superiores para todas as variáveis de crescimento avaliadas, enquanto que as produzidas em espuma fenólica apresentaram os piores resultados. 
Já Matias et al. (1999), avaliando cinco substratos diferentes (fibra de coco sem lavar, fibra de coco lavada, vermiculita, matéria orgânica comercial e espuma fenólica na produção de mudas de alface concluíram que em termos gerais, tanto a matéria orgânica quanto a espuma fenólica mostram-se como o substrato mais adequado para o cultivo hidropônico, principalmente aos onze dias após o início da germinação, enquanto que a matéria orgânica comercial mostra-se melhor como substrato para a produção de mudas para cultivo convencional, em solo. Esses resultados estão de acordo com a proposta do IAC para a utilização de espuma fenólica na produção de mudas pelos produtores que trabalham com hidroponia (Furlani, 1998) .

\section{CONCLUSÕES}

Conclui-se que nas condições em que foi conduzido o experimento que a cultivar Jullie apresentou maior massa fresca da parte aérea do que a cultivar TPC e que no número de folhas a "TPC" se destacou em relação à "Jullie" e que os sistemas de cultivos não influenciaram no rendimento das cultivares.

\section{REFERÊNCIAS}

BASSO, E. N.; BERNARDES, L.J.L. Hidroponia: técnicas de implantação comercial do cultivo de alface. Piracicaba, 1993. 49 p. (Apostila).

BORNER, H. R. Produção de mudas de hortaliças. Guaíba: Agropecuária, 1999. 187 p.

CASTELLANE, P.D.; ARAÚJO, J.A.C. Cultivo sem solo - hidroponia. Jaboticabal: FUNEP/UNESP, 1994. $43 \mathrm{p}$.

FAQUIN, V.; FURTINI NETO, A.E.; VILELA, L.A.A. Produção de alface em hidroponia. Lavras: UFLA, 1996. 50p.

FERREIRA, D.F. Análises estatísticas por meio do Sisvar para Windows versão 4.0. In: REUNIÃO ANUAL DA REGIÃO BRASILEIRA DA SOCIEDADE INTERNACIONAL DE BIOMETRIA. 45., 2000, São Carlos. Anais... São Carlos: UFSCar, 2000. p. 255258.

FILGUEIRA, F.A.R. Novo manual de olericultura. 3. ed. Viçosa: Ed. UFV, 2008. 21p.
FURLANI, P.R. Cultivo de alface pela técnica de hidroponia NFT. Campinas: Instituto Agronômico, 1995. 18 p. (Documentos IAC, 55).

FURLANI, P. R. Instruções para o Cultivo de Hortaliças de Folhas pela Técnica de Hidroponia NFT. Campinas: Instituto Agronômico, 1998. 30 p. (Boletim Técnico, 168).

FURLANI, P. R.; SILVEIRA, L.C.P.; BOLONHEZI, D.; FAQUIN, V. Cultivo hidropônico de plantas: parte 3 - Produção de mudas para hidroponia. 2009. Disponível em: $<$ http://www.infobibos.com/Artigos/20092/hidr oponiap3/index.htm>. Acesso em: 17 jun. 2014.

HORNER, M. C.; PEIL, R, M.; FERREIRA, A. A. F.; STRASSBURGER, A. S.; NICOLLETI, D. R. Avaliação do Crescimento de mudas para o cultivo sem solo do tomateiro em diferentes substratos. Horticultura Brasileira, Brasília, v. 21, n. 2, p. 280, 2003. Suplemento 1.

MATIAS, G. C. S.; COMETTI, N. N.; GÓMEZ, G. P.; ROCHA, J. D. S. Avaliação de substratos comerciais para a produção de mudas de alface. Horticultura Brasileira, Brasília, v.17, n.2, p.159162, 1999.

MORETTI, C. L.; MATTOS, L. M. Processamento mínimo de alface crespa. Brasília: EMBRAPA, 2006. 7 p. (Comunicado Técnico, n. 36).

PAIVA, M. C. Produção de hortaliças em ambiente protegido. Cuiabá: Sebrae-MT, 1998. 78p.

QUEIROZ, S. O. P.; TESTEZLAF. R.; MATSURA, E. E. Avaliação de equipamentos para a determinação da condutividade elétrica do solo. Irriga, Botucatu, v.10, p. 279-287, 2004.

RESH, H.M. Cultivos hidroponicos: nuevas técnicas de producción. 4. ed. Madrid: MundiPrensa, 1997. 378p.

SANTIN, M. M.; SANTOS, H. S.; SCAPIM, C. A.; BRANDÃO, B. M. S.; BRANDÃO FILHO, J. U. T.; CALEGAR, O.; SANTOS, A. J. A.; SANTOS, I. A. Relação entre substratos e métodos de aplicação de solução nutritiva na produção de mudas e a posterior resposta produtiva da beterraba. Acta Sci. Agron. Maringá, v. 27, n. 3, p. 423-432, 2005. 
SCOTT, A.J.; KNOTT, M. A. A cluster analysis method for grouping means in the analysis of variance. Biometrics, v. 30, n. 2, p. 507-512, 1974. https://doi.org/10.2307/2529204

SOUZA, S. R.; SALDANHA, C. S.; FONTINELI, Y. R.; ARAÚJO NETO, S. E.; KUSDRA, J. F. Produção de mudas de alface em sistema floating sob tela de sombreamento e cobertura plástica. Revista Caatinga, v. 20, n. 3, p. 191-195, 2007.

SOUZA, J.C. A.; SOUZA, R. C. J.; COLLICCHIO, E.; GOMES, L. A. A.; SANTOS, H. S. Instruções práticas para construção de estufas "modelo Ana Dias". Lavras: UFLA: 1994. 22P. (Circular Técnica, 17).

Recebido para publicação em 08/01/2018

Revisado em 27/03/2018

Aceito em 02/04/2018 